\title{
Distal chevron osteotomy versus different operative procedures for hallux valgus correction: a meta-analysis
}

\author{
Pablo Clemente ${ }^{1}$, Gonzalo Mariscal ${ }^{2^{*}}$ (1) and Carlos Barrios ${ }^{2}$
}

\begin{abstract}
Introduction: Distal chevron osteotomy is commonly used for the operative treatment of hallux valgus (HV). However, there are several operative procedures that can be used to treat HV. The aim of this meta-analysis was to compare the efficacy of distal chevron osteotomy with different operative procedures.

Materials and methods: A systematic search was conducted using the MEDLINE and EMBASE databases to identify randomized clinical trials (RCTs). The variables were radiological (hallux metatarsal phalangeal angle [HVA] and intermetatarsal angle [IMA]) and clinical (American Orthopaedic Foot \& Ankle Society Score [AOFAS]). Heterogeneity was assessed with chi $^{2}$ and $l^{2}$ statistics. A random effects model was used for significant heterogeneity. Publication bias was evaluated with funnel plots.
\end{abstract}

Results: Ten studies involving 985 patients were evaluated in the meta-analysis. Distal chevron osteotomy was associated with a mean IMA correction $2.18^{\circ}$ greater than the scarf procedure $(\mathrm{MD}-2.18 ; 95 \% \mathrm{Cl}-3.67,-0.69 ; p=0.004$; $P^{2}=0 \%$ ). In addition, the proximal chevron was associated with a mean IMA correction $1.08^{\circ}$ greater than the distal chevron (MD $\left.-1.08 ; 95 \% \mathrm{Cl}-1.86,-0.29 ; p=0.007 ; P^{2}=0 \%\right)$. The AOFAS assessment showed an overall advantage of 3.2 points in favor of the Lingdren group compared with distal chevron osteotomy (MD 3.20; 95\% Cl 0.37, 6.04; $p=0.03 ; 1^{2}=0 \%$.

Conclusions: Our findings indicate that distal chevron osteotomy provides a greater HVA correction than scarf osteotomy, and proximal chevron provides a larger IMA correction than distal chevron osteotomy. Lingdren osteotomy provides a greater AOFAS correction than distal chevron osteotomy.

Level of evidence: Level I, meta-analysis.

Keywords: Chevron osteotomy, Hallux valgus, Distal chevron, Meta-analysis

\section{Introduction}

Hallux valgus (HV) is the most common forefoot pathology in adults. There is also a family history, with a higher prevalence in females [1]. HV is a complex progressive triplanar deformity of the forefoot, characterized by

\footnotetext{
*Correspondence: gonzalo.mariscal@mail.ucv.es

${ }^{2}$ Institute for Research on Musculoskeletal Disorders, School of Medicine and Health Sciences, Valencia Catholic University, Quevedo 2, 46001 Valencia, Spain

Full list of author information is available at the end of the article
}

valgus phalangeal deviation, varus angulation of the first metatarsal, and lateral displacement of the sesamoids and extensor tendons. The initial sign is the formation of a medial bony prominence (bunionette) at the first metatarsophalangeal joint (MTP) [2]. The factors associated with the development of $\mathrm{HV}$ are multiple, but still unclear.

Mann and Coughlin classified HV into three types according to the hallux valgus angle (HVA) and the intermetatarsal angle (IMA): mild $\left(\mathrm{HV}<20^{\circ}\right.$, 
IMA $<11^{\circ}$ ), moderate $\left(\mathrm{HV} 20^{\circ}-40^{\circ}\right.$, IMA $\left.11^{\circ}-16^{\circ}\right)$ and severe $\left(\mathrm{HV}>40^{\circ}, \mathrm{IMA}>16^{\circ}\right)[3]$.

Several operative procedures have been described for the correction of HV. Conventionally, proximal and distal metatarsal osteotomies are used for severe or mild-moderate deformities, respectively [3, 4]. Distal chevron osteotomy alone or combined with other procedures has also shown improved radiological results and patient satisfaction [5]. The most serious adverse event associated with distal chevron osteotomy was osteonecrosis due to disruption of the blood supply to the metatarsal head. However, most studies supporting increased correction and functionality with distal chevron osteotomy have low evidence (level IV) [4]. Distal chevron osteotomy is a commonly used method for the correction of HV. ${ }^{7}$ There are several studies that compare it with other surgical techniques in isolated form [5-14].

The objective of this meta-analysis was to evaluate the optimal operative approach in hallux valgus. This metaanalysis examined different surgical options, comparing the clinical and radiological efficacy of the distal chevron osteotomy with other operative procedures, and thereby providing level I evidence for the surgical treatment of HV.

\section{Material and methods \\ Search strategy and study selection}

The meta-analysis was carried out following the PRISMA criteria (Preferred Reporting Items for Systematic Reviews and Meta-Analyses) [15] (Fig. 1). A bibliographic search was carried out in PubMed, EMBASE, Scopus, and the Cochrane Collaboration Library. The following search terms were used: (1) hallux abducto valgus; (2) randomized clinical trial; and (3) treatment. The search was limited to studies published in the English language. The inclusion criteria were (1) randomized trials in the English language comparing operative techniques in patients with HV; (2) use of the distal chevron osteotomy in one of the study arms; and (3) posttreatment HVA and IMA data and AOFAS scores as the main outcome variables in each study. If more than one study reported data for the same sample, only the most recent and complete one was evaluated.

The results of the selection process are shown in Fig. 1. A total of 94 studies classified as randomized clinical

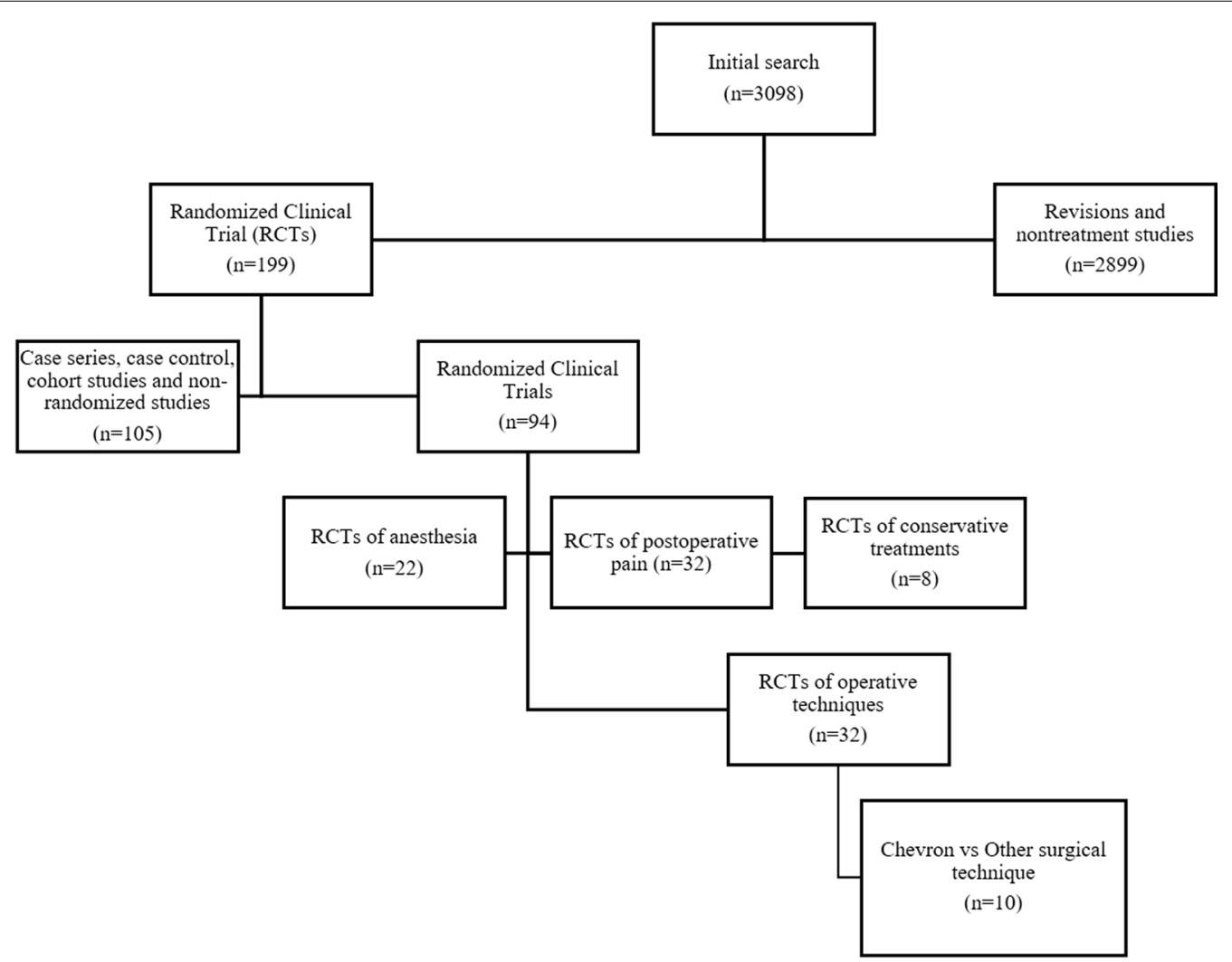

Fig. 1 Study selection flow diagram (Preferred Reporting Items for Systematic Reviews and Meta-Analysis): The PRISMA diagram showing the exclusion and inclusion strategy of randomized clinical trials on distal chevron osteotomy of hallux valgus 
trials on operative techniques were identified using the selected databases. After the first screening, 32 randomized clinical trials related to operative treatment were observed. Sixty-two studies were excluded because they were related to preoperative anesthesia $(n=22)$ postoperative pain control $(n=32)$, or conservative treatments $(n=8)$. Finally, 10 studies comparing chevron distal osteotomies with other surgical techniques were selected.

\section{Data extraction}

The following baseline data were collected: intervention, number of patients, \% of men, mean age, and followup (months). The study variables were divided in two groups: clinical parameters (American Orthopedic Foot and Ankle Score, AOFAS) and radiological measurements (hallux metatarsal phalangeal angle [HVA] and intermetatarsal angle [IMA] between the first and second radius).

\section{Assessment of study quality}

The quality of RCTs was evaluated in accordance with Review Manager (RevMan) version 5.3 (The Nordic Cochrane Centre, The Cochrane Collaboration, Copenhagen, 2014) software to assess for the risk of bias. If there was a conflict between the two reviewers, a third reviewer was consulted to arrive at a decision. The evaluation methods consisted of the following steps: random sequence generation, allocation concealment, blinding, incomplete outcome data, and selective outcome reporting (Fig. 2).

\section{Statistical analysis}

The meta-analysis was performed using the Review Manager 5.4 software package provided by the Cochrane Collaboration. For dichotomous variables, odds ratios with a confidence interval (CI) of $95 \%$ were calculated. The weighted mean difference (WMD) and the $95 \%$ CI were calculated for the continuous variables. Heterogeneity was checked with both the chi ${ }^{2}$ and the $I^{2}$ test. $I^{2}$ varies from 0 to $100 \%$, considering the values of 25,50 and $75 \%$ as low, moderate and high heterogeneity, respectively. A fixed effects model was adopted if there was no statistical evidence of heterogeneity, and a random effects model was adopted if significant heterogeneity was observed. Publication bias was evaluated using the funnel plot diagrams.

\section{Results}

\section{Baseline data}

The main characteristics of the 10 included studies are shown in Table 1 [5-14].The sample sizes ranged from 60 to 136 patients, with a total of 985 patients. The average age of the patients varied from 32.7 to 54 years.

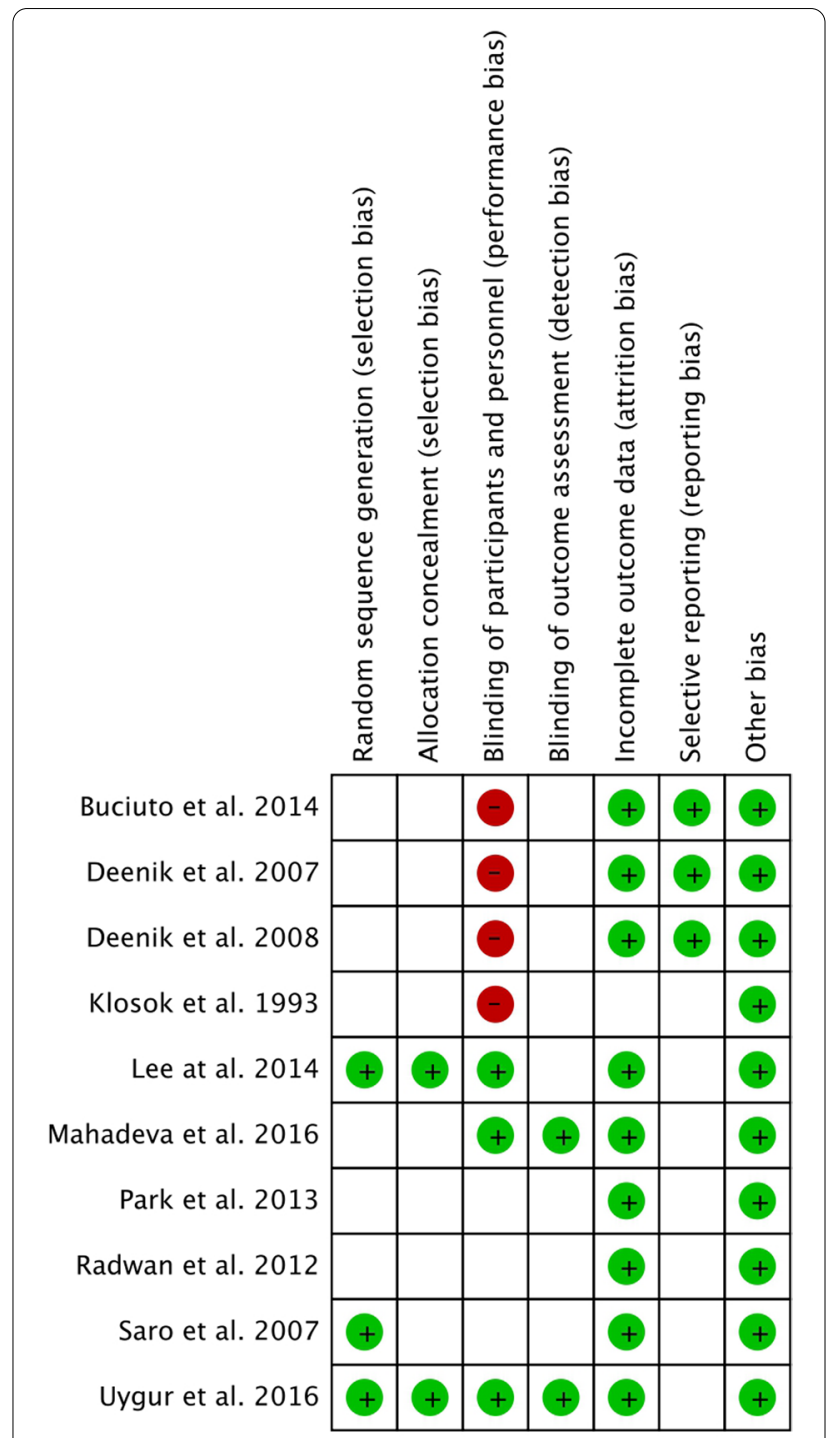

Fig. 2 Risk of bias summary (Green: low risk; white: unknown risk; red: High risk)

Stratification by gender was reported in eight studies, with a percentage of males ranging from 0 to $13.7 \%$. The patients had a follow-up between 12 and 56.4 months.

\section{Radiological outcomes}

The hallux valgus angle (HVA) was described in 11 studies. Four were excluded-one for not providing the standard deviation and three for being isolated noncomparable studies. Seven studies were included: three related to the scarf technique, with 341 patients; two studies compared distal Chevron osteotomy with the Lindgren technique, with 156 patients; and two studies comparing proximal and distal chevron osteotomies, with 202 patients. 


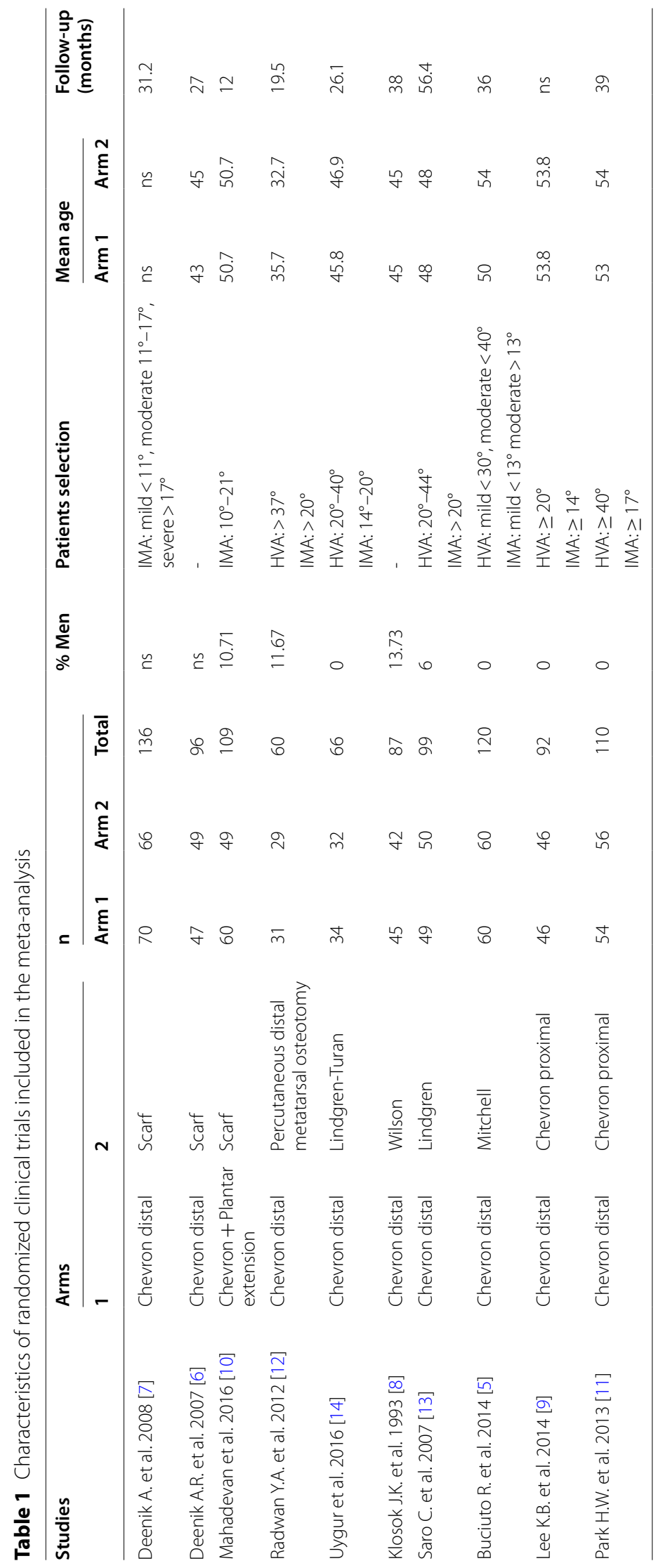


The results regarding the change between HVA showed a greater reduction in the distal chevron osteotomy when compared with the scarf technique (MD-2.18, 95\% $\mathrm{CI}-3.67,-0.69, p=0.004, I^{2}=0 \%$ ) (Fig. 3a). There were no significant differences in HVA when the Lindgren and distal chevron techniques were compared (MD 0.82, 95\% CI $-3.62,5.26, p=0.72, I^{2}=79 \%$ ) (Fig. 3b). No significant differences were observed between the proximal and distal chevron osteotomies (MD 1.26, 95\% CI-0.67, 3.19, $p=0.2, I^{2}=0 \%$ ) (Fig. 3c). The funnel plot diagrams did not show the existence of publication bias (Fig. 4).

The intermetatarsal angle (IMA) was described in 10 studies, of which three were excluded because they were isolated, non-comparable studies. Seven studies were included: three, with a total of 341 patients, dealt with the scarf technique; two studies, with a total of 156 patients, compared distal Chevron osteotomy with the Lindgren technique; and two studies compared the proximal and distal chevron osteotomies.

The results regarding pre- and postoperative IMA values did not show significant differences between the distal chevron and scarf osteotomies (MD $-0.77,95 \%$
CI 1.89, 0.35, $p=0.18, I^{2}=75 \%$ ) (Fig. 5a). No significant differences were observed between the Lindgren and distal chevron techniques (MD 1.14, 95\% CI-0.63, 2.91, $p=0.21, I^{2}=81 \%$ ) (Fig. 5b). Finally, a greater reduction in the IMA was observed in proximal chevron than in distal chevron osteotomies (MD - 1.08, 95\% CI 0.29, 1.86, $p=0.007, I^{2}=0 \%$ ) (Fig. 5c). The funnel plot diagrams did not show the existence of publication bias (Fig. 6).

\section{Clinical outcomes}

The AOFAS scale was described in seven studies. Three studies were excluded, one due to lack of information on the standard deviation and two because they were isolated non-comparable studies. Four studies were included. Two studies, with a total of 156 patients, compared distal chevron osteotomy with the Lindgren technique, and two studies, with a total of 202 patients, compared distal chevron with proximal chevron osteotomy.

The AOFAS before and after surgery showed a significantly improvement in the Lindgren technique compared with distal chevron (MD 3.20, 95\% CI: 0.37,

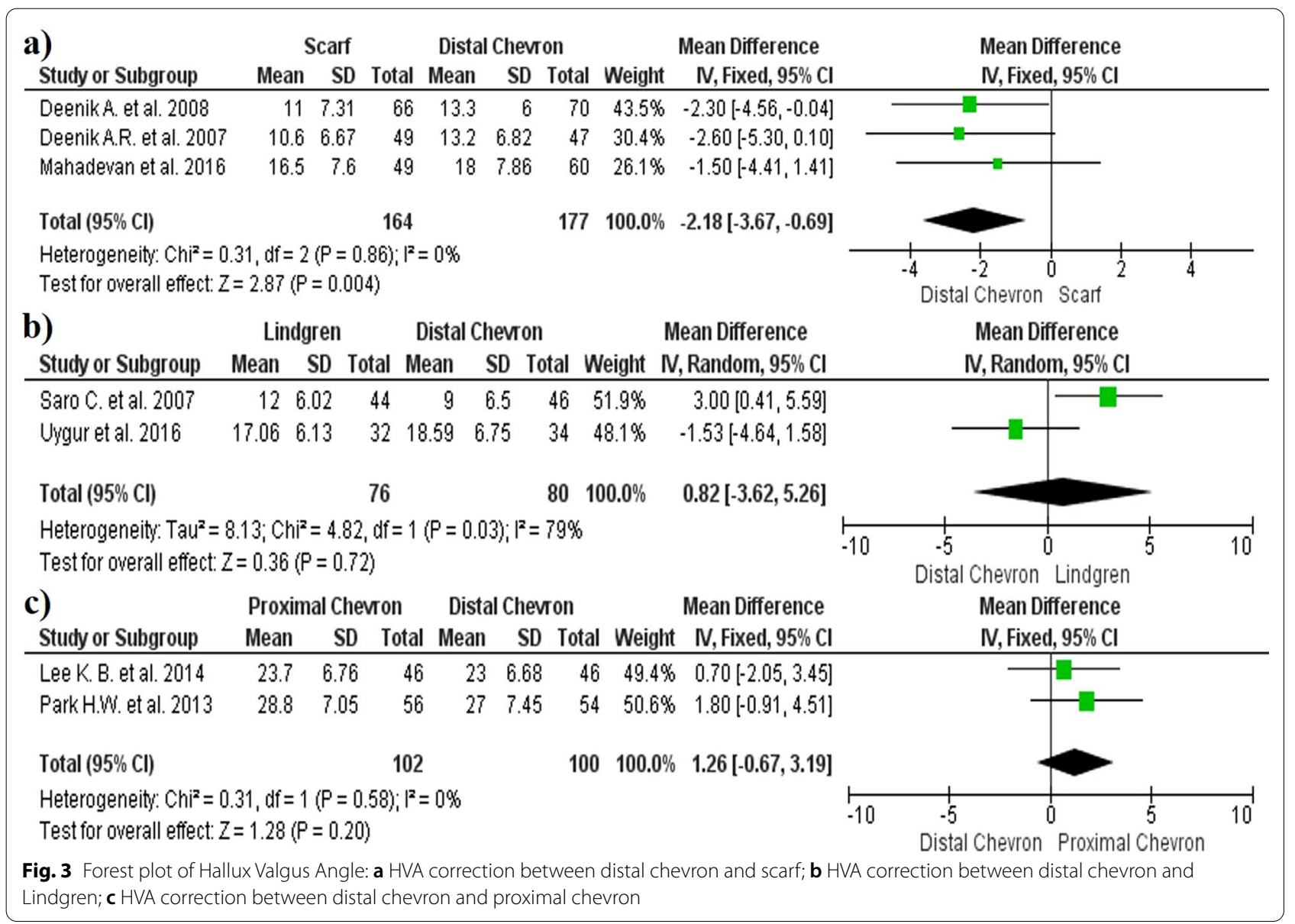



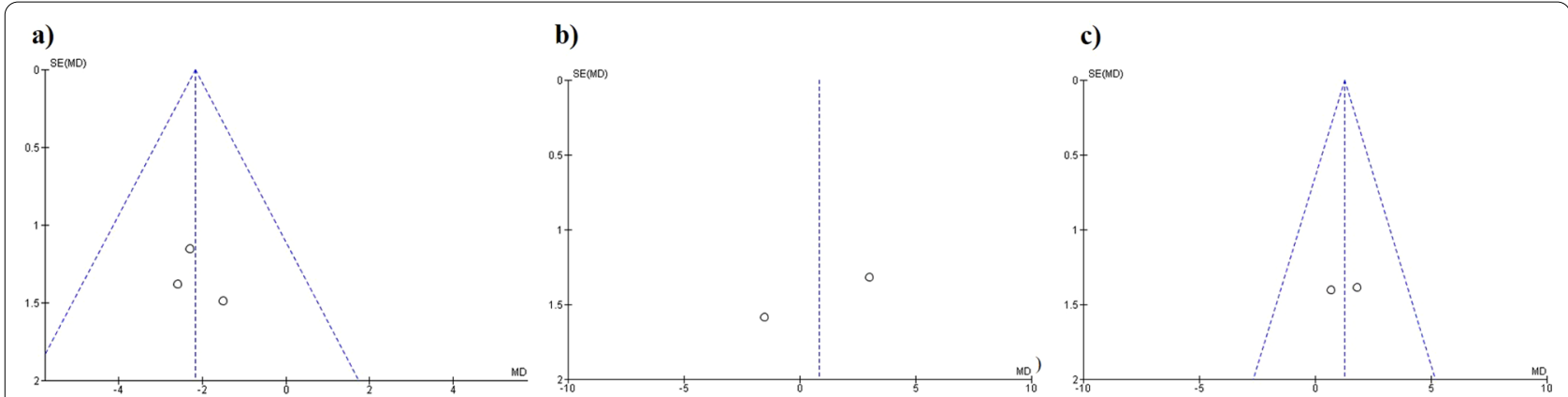

Fig. 4 Hallux Valgus Angle funnel plot diagrams: a Distal chevron versus scarf; b Distal chevron versus Lindgren; c Distal chevron versus proximal chevron

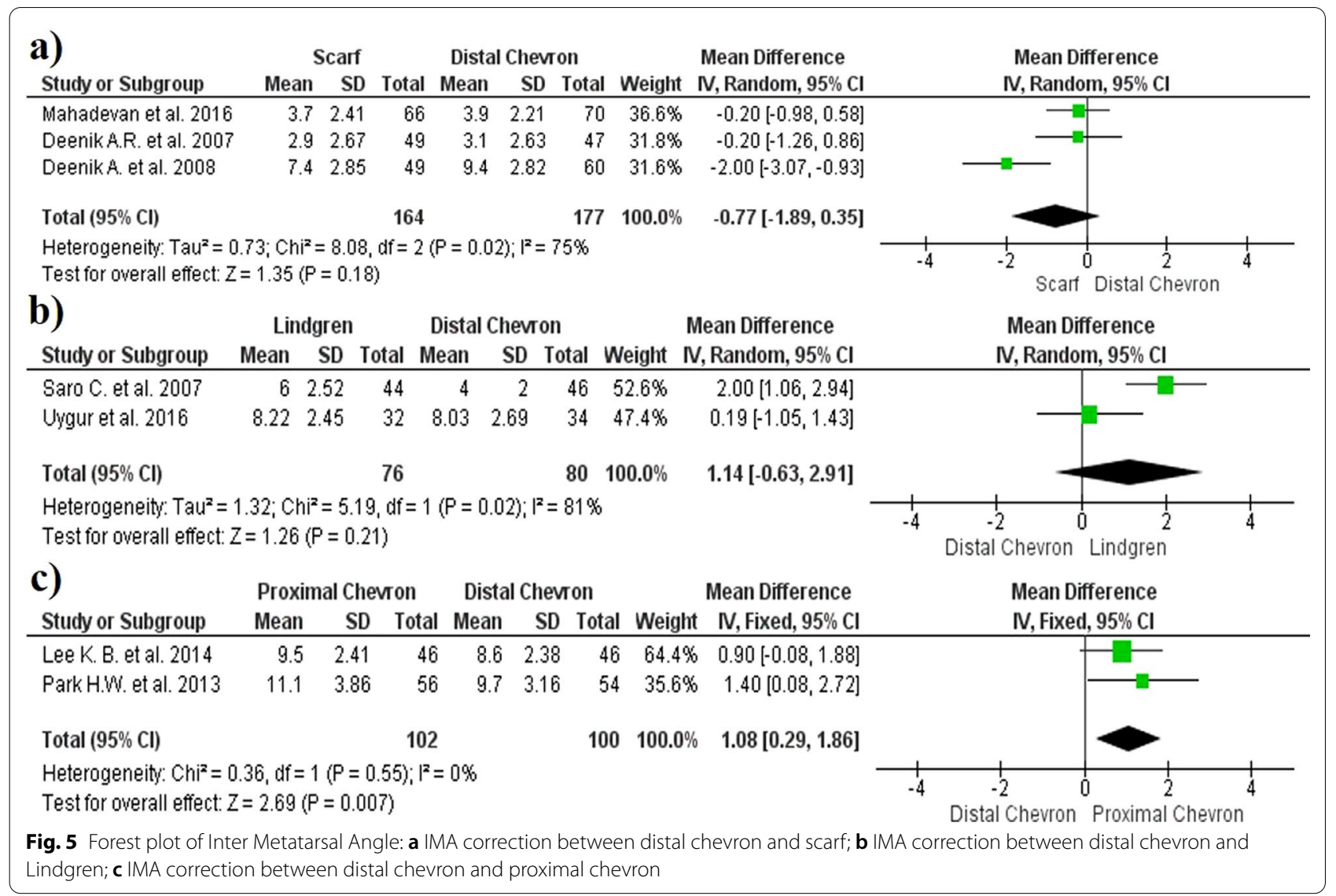

6.04, $p=0.03, I^{2}=0 \%$ ) (Fig. 7a). No significant differences were observed between the proximal and distal chevron (MD 0.96, 95\% CI $-1.85,3.77, p=0.5$, $I^{2}=0 \%$ ) (Fig. 7b). The funnel plot diagrams did not show the existence of publication bias (Fig. 8).

\section{Discussion}

The purpose of this study was to compare the efficacy of distal chevron osteotomy to that of other operative techniques for correcting to hallux valgus in different 

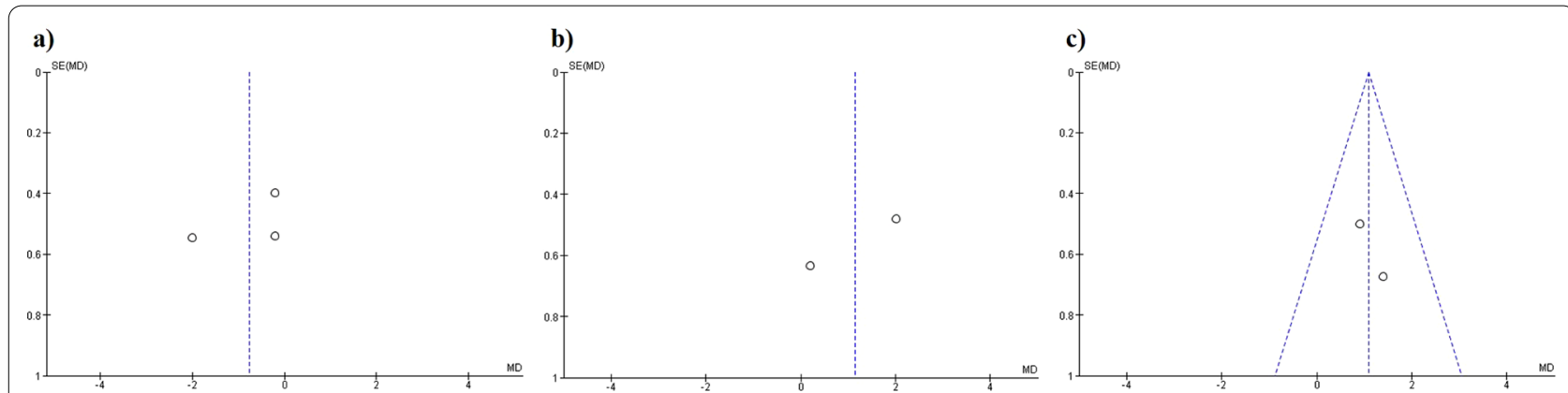

Fig. 6 Inter Metatarsal Angle funnel plot diagrams: a Distal chevron versus scarf; b Distal chevron versus Lindgren; c Distal chevron versus proximal chevron

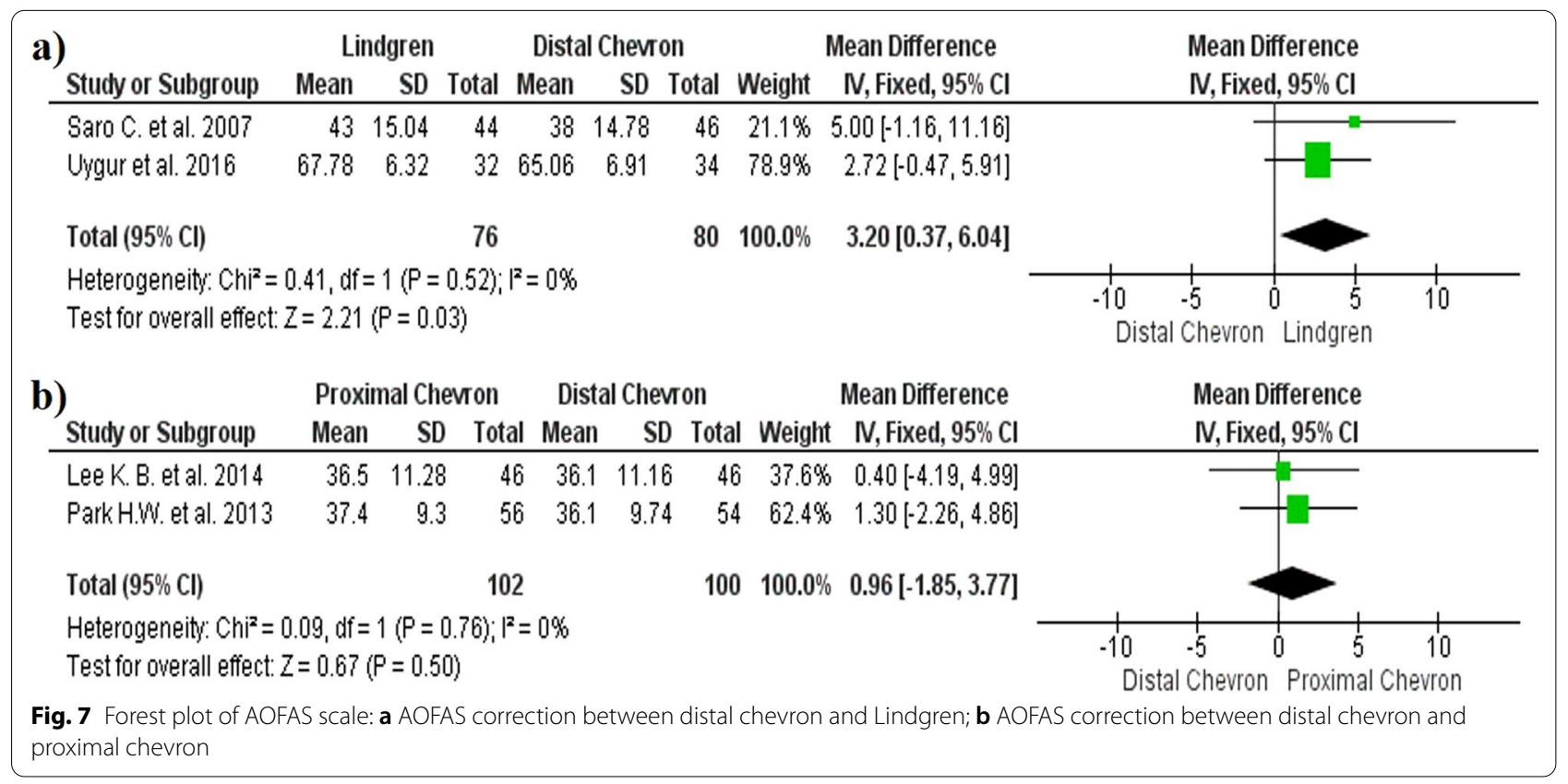

randomized studies. We used radiological parameters (HAV and IMA) and AOFAS as a clinical measure.

Regarding the radiological results, the reduction in the HVA was higher with the distal chevron osteotomy than the scarf technique. In the three included studies, the mean difference in HVA correction was $2.18^{\circ}$ in favor of distal chevron osteotomy. A recent randomized study comparing percutaneous distal chevron osteotomy with scarf osteotomy showed similar results, with a mean correction of $2.7^{\circ}$ higher in distal chevron osteotomy [16]. When the distal chevron technique (spread plantarly) was compared with the scarf technique, both techniques showed similar AHV corrections with respect to our study, with $1.1^{\circ}$ in favor of the scarf technique. There are a few studies in the literature comparing distal chevron and scarf that have a I-II evidence level. There are case series of both techniques, but all of them have a III-IV evidence level, and are therefore not comparable to this meta-analysis. A systematic review of these articles was reported in 2012, but did not include the HVA outcome [18]. Among the case series of scarf osteotomy, corrections of the HVA of $25^{\circ}$ on average have been published, always associated with Akin phalanx osteotomy [19]. For chevron osteotomy, large HVA corrections of more than $30^{\circ}$ have also been described [20].

In this meta-analysis, the mean correction of HVA with distal chevron was $23.22^{\circ}$, slightly higher than the $17.1^{\circ}$ described by Schuh et al. in a systematic review of proximal osteotomies, including 446 proximal chevron osteotomies. 


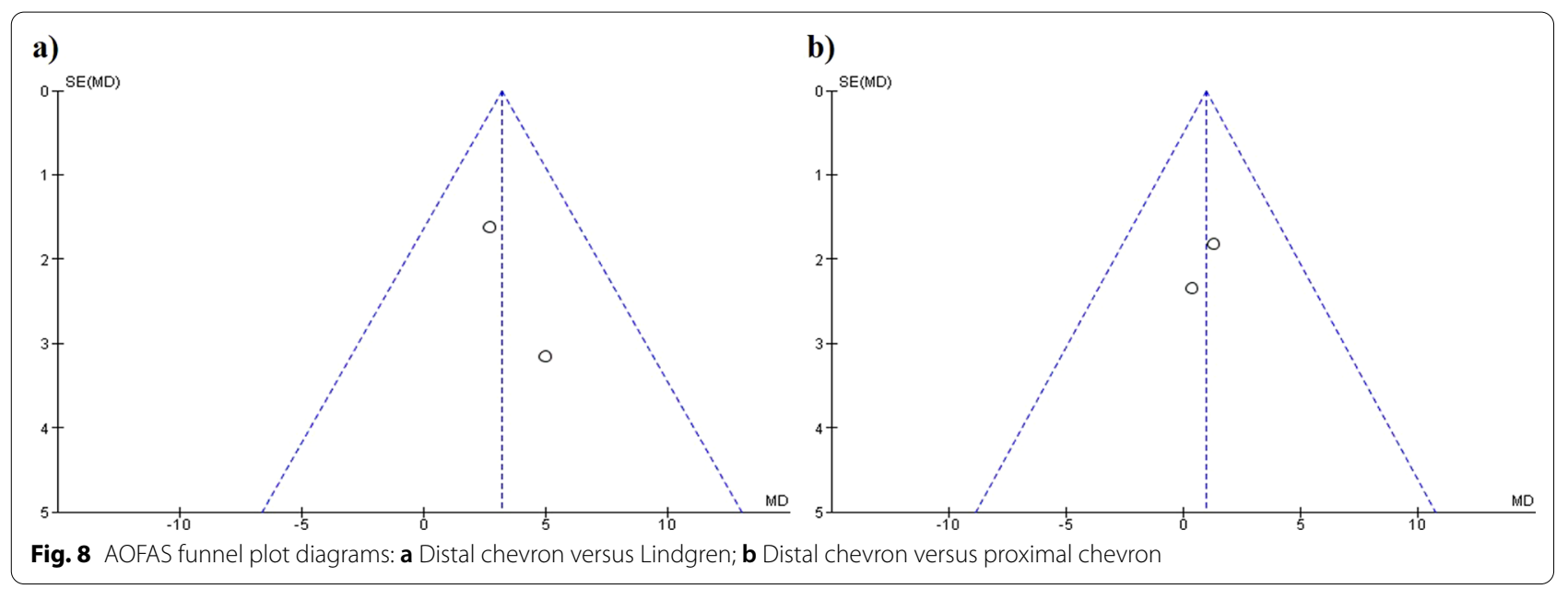

When it came to the IMA, the proximal chevron osteotomy showed a greater reduction than the distal chevron, so the proximal chevron osteotomy could be the first option if a greater radiological correction is required. In contrast, there was no difference between distal chevron osteotomy and Lingdren or scarf osteotomy. There was only one meta-analysis that compared distal chevron and scarf osteotomy, which was published by Smith et al. in 2012. This study made a compilation of 31 studies with 1351 patients. The researchers' results showed a significant reduction of $0.88^{\circ}$ in favor of scarf osteotomy [18]. Our study showed no significant difference in IMA correction between these two procedures. According to the literature, scarf osteotomy showed a greater correction of IMA [19].

Lateral translation in both osteotomies is limited, as bone contact is essential for adequate fixation. In X-rays, the appearance of the first metatarsal after chevron osteotomy is different from after scarf osteotomy, as the chevron osteotomy shows a valgus deformity in the long axis of the first metatarsal. In scarf osteotomy, the correction of the longitudinal axis is more aligned. However, the biomechanical axis and therefore the IMA are defined by the corrected metatarsal head and not by the shape of the axis [21]. Smith et al. measured the center of the metatarsal head separately from the magnitude of the bunionette. This method of measurement has been found to be the most reliable [22].

However, the radiological measures, both HVA and IMA, are considered to be inaccurate and only include assessments in a single plane. Thus, comparisons between studies should be evaluated carefully [23].

Regarding the AOFAS, Lingdren osteotomy could be chosen over distal chevron osteotomy based on clinical criteria. On the other hand, there is no difference between distal and proximal chevron osteotomies in the improvement of the AOFAS.

When comparing the minimally invasive technique with chevron osteotomy, no short- and long-term differences were found between quality of life scores, radiological parameters and range of motion. Only higher patient satisfaction was observed with the minimally invasive technique at 12 weeks $[24,25]$.

Some of the limitations of this meta-analysis were the sample sizes of the different studies, so there was no subgroup analysis. Regarding quality of life or pain outcomes, only the AOFAS and VAS could be compared. Also, the traditional standard chevron procedure is used for moderate hallux valgus correction. However, in recent years, due to the continuous improvement of the surgical method, it has also been applied to severe hallux valgus. The Chevron surgical approach is under continuous development.

\section{Conclusion}

In conclusion, this meta-analysis considered articles not included in recent meta-analyses. It is also the only metaanalysis to our knowledge that includes an analysis of the publication bias (funnel plot) of each outcome. According to the results of this meta-analysis, Lingdren osteotomy with respect to produces a greater clinical improvement than distal chevron osteotomy, as assessed by the AOFAS scale. Similarly, distal chevron osteotomy produces better clinical results than the scarf technique. Finally, proximal chevron osteotomy achieves a greater reduction in IMA than distal chevron osteotomy.

\section{Abbreviations}

AOFAS: American Orthopaedic Foot \& Ankle Society Score; HV: Hallux Valgus; HVA: Hallux Metatarsal Phalangeal Angle; IMA: Intermetatarsal Angle; MTP: 
Metatarsophalangeal joint; PRISMA: Preferred Reporting Items for Systematic Reviews and Meta-Analyses; WMD: Weighted Mean Difference.

\section{Authors' contributions}

PC, GM and CB provided the study materials, designed the recording and interpretation of clinical trials. PC, GM and CB contributed to the analysis of data. PC, GM and CB contributed to the writing of the first draft of manuscript. $P C, G M$ and $C B$ designed the figures. All authors read and approved the final manuscript.

\section{Funding}

The authors received no financial support for the research, authorship, and/or publication of this article.

\section{Declarations}

\section{Consent for publication}

Not Applicable.

\section{Ethics approval and consent to participate}

This is a meta-analysis and therefore no ethics committee is required.

\section{Informed consent}

This is a meta-analysis, therefore, it does not require an informed consent.

\section{Competing interests}

The authors declare that they have no competing interests.

\section{Author details}

'Department of Traumatology and Orthopedic Surgery, Hospital Doctor Peset, Valencia, Spain. ${ }^{2}$ Institute for Research on Musculoskeletal Disorders, School of Medicine and Health Sciences, Valencia Catholic University, Quevedo 2, 46001 Valencia, Spain.

Received: 12 September 2021 Accepted: 25 January 2022

Published online: 08 February 2022

\section{References}

1. Mann RA, Coughlin MJ. Hallux valgus - etiology, anatomy, treatment and surgical considerations. Clin Orthop Relat Res. 1981;157:31-41.

2. Badekas A, Georgiannos D, Lampridis V, Bisbinas I. Proximal opening wedge metatarsal osteotomy for correction of moderate to severe hallux valgus deformity using a locking plate. Int Orthop. 2013;37:1765-70.

3. Coughlin MJ, Saltzman CL, Anderson RB. Mann's surgery of the foot and ankle. 9th ed. St. Louis: Mosby; 1999.

4. Easley ME, Trnka HJ. Current concepts review: hallux valgus part II: operative treatment. Foot Ankle Int. 2007;28(6):748-58.

5. Buciuto R. Prospective randomized study of chevron osteotomy versus Mitchell's osteotomy in hallux valgus. Foot Ankle Int. 2014;35(12):1268-76.

6. Deenik AR, Pilot P, Brandt SE, van Mameren H, Geesink RGT, Draijer WF. Scarf versus chevron osteotomy in hallux valgus: a randomized controlled trial in 96 patients. Foot Ankle Int. 2007;28:537-41.

7. Deenik A, van Mameren H, de Visser E, de Waal MM, Draijer F, de Bie R. Equivalent correction in scarf and chevron osteotomy in moderate and severe hallux valgus: a randomized controlled trial. Foot Ankle Int. 2008;29(12):1209-15.

8. Klosok JK, Pring DJ, Jessop JH, Maffulli N. Chevron or Wilson metatarsal osteotomy for hallux valgus. A prospective randomised trial. J Bone Jt Surg (Br). 1993;75-B:825-9.

9. Lee KB, Cho NY, Park HW, Seon JK, Lee SH. A comparison of proximal and distal Chevron osteotomy, both with lateral soft-tissue release, for moderate to severe hallux valgus in patients undergoing simultaneous bilateral correction. A prospective randomised controlled trial. Bone Jt J. 2015;97-B(2):202-7.

10. Mahadevan D, Lines S, Hepple S, Wilson I, Harries W. Extended plantar limb (modified) chevron osteotomy for hallux valgus correction: a randomised controlled trial. Foot Ankle Surg. 2016;22:109-13.
11. Park HW, Lee KB, Chung JY, Kim MS. Comparison of outcomes between proximal and distal chevron osteotomy, both with supplementary lateral soft-tissue release, for severe hallux valgus deformity. A prospective randomised controlled trial. Bone Jt J. 2013;95-B(4):510-6.

12. Radwan YA, Mansour AM. Percutaneous distal metatarsal osteotomy versus distal chevron osteotomy for correction of mild-to-moderate hallux valgus deformity. Arch Orthop Trauma Surg. 2012;132(11):1539-46.

13. Saro C, Andrén B, Wildemyr Z, Felländer-Tsai L. Outcome after distal metatarsal osteotomy for hallux valgus: a prospective randomized controlled trial of two methods. Foot Ankle Int. 2007;28(7):778-87.

14. Uygur E, Özkan NK, Akan K, Çift H. A comparison of Chevron and Lindgren-Turan osteotomy techniques in hallux valgus surgery: a prospective randomized controlled study. Acta Orthop Traumatol Turc. 2016;50(3):255-61

15. Liberati A, Altman DG, Tetzlaff J, et al. The PRISMA statement for reporting systematic reviews and meta-analyses of studies that evaluate healthcare interventions: explanation and elaboration. BJM. 2009;339:b2700.

16. Lee M, Walsh J, Smith MM, Ling J, Wines A, Lam P. Hallux valgus correction comparing percutaneous chevron/akin (PECA) and Open scarf/akin osteotomies. Foot Ankle Int. 2017:38(8):838-46.

17. Vopat BG, Lareau CR, Johnson J, Reinert SE, DiGiovanni CW. Comparative study of scarf and extended chevron osteotomies for correction of hallux valgus. Foot Ankle Spec. 2013;6:409-16.

18. Smith SE, Landorf KB, Butterworth PA, Menz HB. Scarf versus chevron osteotomy for the correction of 1-2 intermetatarsal angle in hallux valgus: a systematic review and meta-analysis. J Foot Ankle Surg. 2012;51(4):437-44.

19. Mann RA. Decision-making in bunion surgery. Instr Course Lect. 1990;39:3-13.

20. O'Donnell T, Hogan N, Solan M, Stephens MM. Correction of severe hallux valgus using a basal chevron osteotomy and distal soft tissue release. Foot Ankle Surg. 2010;16(3):126-31.

21. Miller JW. Distal first metatarsal displacement osteotomy. Its place in the schema of bunion surgery. J Bone Jt Surg. 1974;56-A:923-31.

22. Schneider W, Csepan R, Knahr K. Reproducibility of the radiographic metatarsophalangeal angle in hallux surgery. J Bone Joint Surg Am. 2003;85(3):494-9.

23. Nyska M. Principles of first metatarsal osteotomies. Foot Ankle Clin. 2001;6:399-407.

24. Kaufmann G, Dammerer D, Heyenbrock F, Braito M, Moertlbauer L, Liebensteiner M. Minimally invasive versus open chevron osteotomy for hallux valgus correction: a randomized controlled trial. Int Orthop. 2019;43(2):343-50.

25. Kaufmann G, Mörtlbauer L, Hofer-Picout P, Dammerer D, Ban M, Liebensteiner M. Five-year follow-up of minimally invasive distal metatarsal chevron osteotomy in comparison with the open technique: a randomized controlled trial. J Bone Jt Surg Am. 2020;102(10):873-9.

\section{Publisher's Note}

Springer Nature remains neutral with regard to jurisdictional claims in published maps and institutional affiliations.

Ready to submit your research? Choose BMC and benefit from

- fast, convenient online submission

- thorough peer review by experienced researchers in your field

- rapid publication on acceptance

- support for research data, including large and complex data types

- gold Open Access which fosters wider collaboration and increased citations

- maximum visibility for your research: over $100 \mathrm{M}$ website views per year

At BMC, research is always in progress.

Learn more biomedcentral.com/submissions 